The understanding of how proteins are translocated across lipid bilayers is a fundamental question in organelle biogsenesis. Considerable progress has been made in the field of mitochondrial protein topogenesis over the last few years (for recent reviews see Refs 1-6). Recent developments were discussed at a meeting that focused on three aspects of mitochondrial biogenesis: the identification and characterization of the translocation machinery involved in the passage of proteins across the two mitochondrial membranes; protein folding ind the role of heat shock proteins; and the intramitochondrial sorting of proteins.

\section{The outer membrane translocation machinery}

Mitochondrial proteins are imported from the cytosol across the outer membrane $(O M)$ by a multisubunit translocation machinery. The subunits appear to be of similar size in Saccharomyces cerevisiae and Neurospora crassa (Klaus Pfanner, Freiburg, FRG), the two organisms of choice to study this process. In $N$. crassa two import receptors for mitochondrial precursor proteins have been identified: MOM19 and MOM72 (MAS70 in S. cerevisiae). MOM19 is involved in translocation of the majority of mitochondrial precursors, whereas MOM72 is primarily concerned with the binding of the precursor of the ADP/ATP carrier, although it has a low affinity for other precursors. The $65 \mathrm{kDa}$ cytoplasmic domain of MOM72 was shown to contain the binding site for the precursors. It was suggested that the two OM receptors may act as a backup system for each other. The OM translocation pore is composed of MOM38 (ISP42 in S. cerevisiae), MOM30, MOM7 and MOM8.

In a genetic approzch to studying the OM translocation machinery in 5. cerevisiae (Michael Douglas, Chapel Hill, USA), a high-copy suppressor, ISP6, of temperature-sensitive mutants of $15 P 42$ (isp42 $2^{\text {ts }}$ ) has been identified. ISP6 encodes an OM protein that forms a complex with ISP42. Delection of ISP6 produced no apparent phenotype in a wild-type background, but in the isp42ts strain it was lethal. Whether ISP6 is homologous to $N$. crassa MOM7 or MOM8 is not yet clear.

Very little is known about the molecular mechanism of the translocation event across the OM; however, an OM vesicle system has now

\title{
Sorting out mitochondrial proteins
}

Rosemary A. Stuart, Roland Lill and Walter Neupert

been established to study this process. These vesicles can translocate precursor proteins of the $O M$ and of the intermembrane space (IMS) in a receptor-dependent manner (Roland Lill, Munich, FRG).

\section{The inner membrane}

\section{translocation machinery}

Two components of the elusive translocation machinery of the inner membrane (IM) may now have been identified: MPI1 is an essential $S$. cerevisiae protein of the mitochondrial IM, which was identified by a genetic screen developed to isolate mitochondrial import mutants (Michiel Meijer, Amsterdam, The Netherlands). Altogether, 19 recessive nuclear mutations were obtained which, in addition to MPII, identified the SSC1 gene that encodes mitochondrial HSP70, previously shown to be essential for matrix import, and another gene, MPI2. A genetic interaction between MPI2 and SSC1 was observed, suggesting that MPI2 might be a mitochondrial homologue of the bacterial DnaJ protein (see below).

In an elegant biochemical approach, a matrix-targeted precursor protein that was arrested in the IM during translocation was crosslinked to putative components of the translocation machinery (Ute Krieg, Basel, Switzerland). A 45 kDa peripheral IM protein (ISP45) was thus identified. Furthermore, antibodies against IM proteins of $-45 \mathrm{kDa}$ inhibited import of proteins into 'right-side-out' IM vesicles. These antibodies enabled the identification of the $45 \mathrm{kDa}$ protein previously mentioned and of a $\mathbf{4 0} \mathrm{kDa}$ protein (ISP40). Tryptic peptide analysis revealed the latter protein to be identical to MPI1.

\section{Proteln folding and heat shock proteins}

In general, translocation of precursor proteins across membranes is believed to require stabilization of an unfolded conformation of the precursor. In $S$. cerevisiae, it appears that cytosolic HSP70 plays an essential role in this regard: cytosolic HSP70 interacts with YDI1, a eukaryotic homologue of the bacterial pro- tein Dnal, thereby stimulating the ATPase activity of HSP70 (Michael Douglas). It was proposed that cytosolic HSP70 first binds to mitochondrial precursor proteins, then YDJ1 binds to the HSP70-precursor complex, thereby promoting the release of the bound polypeptide in an ATP-dependent manner. Farnesylation of YD|1 appears to mediate its partitioning between the cytosol and subcellular membranes. This might assist in targeting HSP70-precursor complexes to the OM. The importance of YD|1 is supported by the finding that temperature-sensitive mutants of $\mathrm{YD} / 1$ display defects in protein transport to mitochondria (also reported by Mike Yaffe, San Diego, USA).

In mammalian cells, the maintenance of proteins in an unfolded conformation seems to be even more complicated: a presequencebinding factor (PBF) of $50 \mathrm{kDa}$ is required for the import into rat liver mitochondria of precursors with cleavable presequences. It was proposed that PBF may bind to and prevent precursors from folding into an unfavourable conformation for translocation. Rabbit PBF is a hydrophilic protein with acidic domains at the $\mathrm{N}$ - and $\mathrm{C}$-termini that might interact with the positively charged residues of mitochondrial presequences (Masataka Mori, Kumamoto, Japan). A second cytosolic factor, mitochondrial import stimulation factor (MSF) is a heterodimer of $30 \mathrm{kDa}$ and 32 $\mathrm{KDa}$ subunits and stimulates the import of the precursor of the matrix-localized protein adrenodoxin (Tsunco Omura, Fukuoka, Japan). MSF is thought to promote both depolymerization of the oligomeric precursor and unfolding in an ATPdependent manner.

Continuing the theme of protein folding, disappointingly little new information was reported on the action of mitochondrial HSP7O. Furthermore, it seems that the pacemaking studies of mitochondrial heat shock proteins may now have been taken over by studies of chaperones in other subcellular subcompartments. For example, exciting new developments on the understanding of the molecular

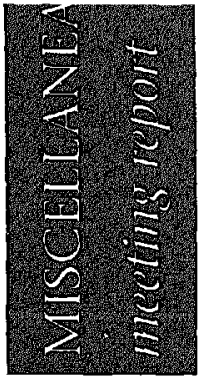

Protein Targeting to Mitochondria. Bayerische Akadernie der Wissenschaften, München, FRG; 15-17 October 1992. Sponsored by the Deutsche Forschungsgemeinschaft (SonderForschungsbereich 184) and the European Science Foundation Network.

The authors are at the Institut für Physiologische Chemie der Universităt München, Goethestrasse 33 , 8000 München 2 , FRG. 
function of GroEL. and TCP1 were reported (F-Ulrich Hartl, SloanKettering, NY, USA; Arthur Horwich, Yale, USA). An IMS protein, SCO1, that appears to be involved in the folding and assembly of cytochrome oxidase subunit II, and possibly of other proteins of this subcompartment, was discussed by Gerhard Rödel (Ulm, FRG). SCO1, a 33 kDa protein, displays limited homology to members of the Dnal family. This interesting observation may be the first evidence for a chaperone in the IMS.

Many mitochondrial precursor proteins require ATP outside the mitochondrion for their import in vitro or else they must be first denatured, for example by urea, to be imported in an ATP-independent fashion. In addition, fusion proteins consisting of mitochondrial targeting sequences fused to cytosolic mouse dihydrofolate reductase do not seem to need cytosolic ATP. Thus, the conformational state of the precursor dictates its requirement for cytosolic ATP for import. By contrast, the requirement for hydrolysis of matrix ATP during protein import cannot be overcome by urea denaturation of the precursor (Gottfriert Schatz, Basel, Switzerland). This observation provoked a discussion on a previous misconception shared by several laboratories, namely the definition of 'ATP-depleted mitochondria'. Previous attempts to deplete the matrix of ATP have probably succeeded in reducing the matrix ATP concentration to below the apparent $K_{m}$ for import of matrix proteins; however, this should not be taken as a criterion for depletion from the matrix of all ATP. The ATP levels remaining after 'depletion treatments' may weil be sufficient to promote import of certain IMS proteins (Walter Neupert, Munich, FRC). It is interesting in this regard that import of the IMS protein cytochrome $c_{1}$ does not appear to require high matrix ATP levels (Cottfried Schatz).

\section{Proteolytic processing of signal sequences}

Once in the mitochondrial matrix, precursor proteins are proteolytically cleaved to remove their matrixtargeting sequences. The matrix processing peptidase, originally termed MPP, is in fact composed of two components: MPP ( $N$. crassa) or MAS2/MIF2 (S. cerevisiae), and PEP, the processing-enhancing protein (N. crassa) or MAS1/MIF1 (S. cere- visiae). Since this nomenclature is rather confusing it was agreed at this meeting to rename the two components $\alpha$-MPP and $\beta-M P P$, respectively.

In an attempt to understand the molecular properties of MPP, a purified system has been used (Michael Brunner, Munich, FRG) in which presequence peptides of cytochrome $b_{2}$ inhibit the processing reaction in a competitive fashion. Since peptides corresponding to various regions of the presequence have different effects in this system, it was proposed that the sequence requirements for mitochondrial import and recognition by MPP are distinct. Studies of MPP from plant mitochondria have revealed a number of unexpected characteristics of the enzyme. In potato mitochondria, MPP activity is exclusively associated with the cytochrome $b c_{1}$ complex of the IM (Udo Schmitz, Berlin, FRG). This is in marked contrast to the situation in S. cerevisiae mitochondria, where the enzyme is completely soluble in the matrix. Interestingly, in $N$. crassa only $\beta$-MPP is associated with the cytochrome $b c_{1}$ complex.

The mitochondrial intermediate peptidase (MIP) is responsible for the removal of octapeptides remaining on some precursors after their cleavage by MPP in the matrix. MIP activity has previously been observed in rat, $N$. crassa and $S$. cerevisiae mitochondria. Rat liver MIP is a protein of $75 \mathrm{kDa}$ that requires divalent cations for activity and can be competitively inhibited by specific octapeptides (Frantisek Kalousek, Yale, USA). A putative yeast homologue of MIP was found on the yeast chromosome III when the complete sequence was reported recently. Perhaps deletion of this gene will shed light on the function of the octapeptide sequences, which so far can only be guessed.

\section{Three routes to the \\ intermembrane space}

Several pathways have been described for the sorting of precursors to the various submitochondrial compartments. In the case of targeting to the IMS, at least three pathways exist. One of these is the pathway taken by cytochrome $c$ haem lyase (CCHL), a peripheral IM protein that is imported directly across the OM, facilitated by the translocation machinery of this membrane (Roland Lill). The nature of the driving force for this process remains enigmatic, as no requirement has been found for ATP or a membrane potential. Like $\mathrm{CCHL}$, many proteins present in the IMS are synthesized without cleavable presequences. It is currently an open question whether all these proteins follow the same import pathway or have similar targeting information.

A second import pathway to the IMS is that taken by cytochrome $c$, which follows a unique route. Mark Dumont (Rochester, USA) discussed the possibility that this precursor in S. cerevisiae simply transverses the outer membrane and then becomes trapped inside the IMS by binding to $\mathrm{CCHL}$ there. In addition to $\mathrm{CCHL}$, another component may be involved in cytochrome $c$ biogenesis in S. cerevisiae, namely the product of the $\mathrm{CYC} 2$ gene, an IM protein.

The targeting mechanism to the IMS of precursors, such as cytochrome $b_{2}$ and cytochrome $c_{1}$, that contain bipartite signal sequences remains a controversial issue. The most N-terminal signal of these precursors is a matrix-targeting sequence, and this is followed by a second signal that has been termed the sorting signal. The manner in which this sorting signal operates in targeting precursors to the IMS is a source of disagreement. One view is that, because of its hydrophobicity, it serves as a 'stop-transfer' domain that arrests the imported protein in the IM. Subsequent sorting to the IMS could then occur by lateral diffusion in the $\mathbb{M}$. The contrasting view maintains that these proteins are imported along the general import pathway into the matrix from where they embark on the export pathway across the IM to the IMS. This latter model has been termed 'conservative sorting' because the mechanism of sorting is believed to reflect the route of protein export from bacteria, which may be the evolutionary ancestors of mitochondria.

In support of the 'stop-transfer' hypothesis, Ben Glick (Basel, Switzerland) discussed several experiments that suggest IMS proteins do not pass through the matrix. By using a derivative of sytochrome $b_{2}$ that was biotinylated on the sorting domain he showed that the now hydrophilic sorting domain failed to function as a stop-transfer domain and the protein was targeted to the matrix. Once in the matrix, the biotin group was released due to the reducing environment, but the protein was 
not then exported. This result suggests that the sorting domain does not function as an export signal; however, it was unclear whether the precursor in the matrix remained competent for membrane translocation in these experiments.

According to the 'conservative sorting' model, sorting signals are functionally homologous to bacterial leader sequences in promoting the export of the proteins from the matrix across the inner membrane. In bacterial leader sequences, positive charges preceding the hydrophobic domain are important for export. Mutations in a similar sequence motif found in the sorting signals of cytochrome $b_{2}$ and $c_{1}$ should, according to the "conservative sorting' model, result in the accumulation of intermediates in the matrix. To test this hypothesis, Elisabeth Schwarz (Munich, FRG) performed a mutational analysis of these positively charged residues of the IMS sorting signal in the cytochrome $b_{2}$ presequence, and found that the mutated proteins accumulated in the matrix as intermediate-sized forms. These results were interpreted to suggest that the sorting signal functions in a similar manner to prokaryotic leader export signals and that it is recognized in a highly specific manner by so far unknown components of the matrix.

\section{Protelns involved in sorting to the Intermembrane space}

It had previously been suggested that HSP60 plays a role in the sorting of cytochrome $b_{2}$ from the matrix to the IMS, by modulating the folding of the protein in the matrix. To address this possibility further, Richard Hallberg (Syracuse, USA) created a series of conditional lethal $h s p 60$ mutants to determine whether depletion of HSP60 could affect the topogenesis of the IMS proteins cytochromes $c_{1}$ and $b_{2}$. No dramatic effect of HSP60 depletion on the maturation of cytochrome $b_{2}$ was observed, suggesting HSP60 is not involved in the sorting of such precursors. Due to the complexity of the system, a more extensive analysis will be needed.

In another approach to define the components involved in the sorting of IMS proteins, S. cerevisiae petite mutants, which are defective in the assembly of the mitochondrionencoded cytochrome oxidase subunit II, were analysed for a simultaneous deficiency in the assembly of cytochromes $b_{2}$ and $c_{1}$ (lodi Nunnari, San Francisco, USA). Three such mutants were detected; one of them led to the cloning of a gene encoding a $30 \mathrm{kDa}$ mitochondrial protein with no apparent homology to other proteins and of no known function. It will be interesting to see if this component plays a role in the sorting of precursors to the IMS.

In an attempt to study the putative export of IMS proteins, inverted inner membrane vesicles of rat liver mitochondria were used (Syozo Tuboi, Yamagata, Japan). Transport of preproalbumin into such vesicles was reported. It will be interesting to see whether authentic mitochondrial precursors can also be translocated in this system.

\section{Assembly of inner membrane proteins}

The question of how nucleusencoded proteins are assembled into the IM may be as difficult as it is interesting. The exact topology of only a few of these proteins are known and tools to study the details of insertion and folding in the membrane are rather limited. Promising approaches were reported for cytochrome oxidase subunit Va (Michael Cumsky, Irvine, USA) and $F_{0}$ ATPase subunit 8 (Phillip Nagley, Monash, Australia). The latter is in fact a mitochondrial gene product, but in these experiments a construct encoding a precursor containing a mitochondrial matrix-targeting senuence was expressed in the nucleus.

A different approach to understanding the rules governing insertion into the IM is a statistical analysis of the distribution of positive charges in translocated and nontranslocated segments (Gunnar von Heijne, Stockholm, Sweden). The 'positive-inside' rule was initially formulated for bacterial membrane proteins and proteins of the eukaryotic endoplasmic reticulum membrane. It seems to hold true also for the mitochondrial $I M$, and is particularly applicable to the distribution of positive charges in mitocitondrial gene products. Nucleus-encoded proteins also have many positively charged residues in the matrix space, although there are clearly many exceptions to this rule. Apparently, if there is a message from the distribution of positive charges with regard to the pathways of membrane insertion, it is difficult to decipher.

Similarly, the rules that govern insertion of proteins into the $O M$ are largely obscure and a detailed analysis of the signals responsible are just beginning (Gordon Shore, Montreal, Canada). It may prove easier to understand insertion into the $O M$ because the events involved in targeting nucleus-encoded proteins to the $\mathrm{OM}$ seem to be less complex than those for the IM.

\section{New directions in} mitochondrial blogenesis

A number of new aspects of mitochondrial membrane biogenesis were also raised at this meeting. The task of understanding how precursors become assembled into multimeric complexes, especially those that require coordination of expression of subunits from both the nuclear and mitochondrial genomes is being undertaken by Hanns Weiss (Düsseldorf, FRG) and Bernard Trumpower (Dartmouth, USA) with the energy-transducing complexes I and III, respectively, of the respiratory chain. The biophysics of protein insertion into the lipid bilayer is also largely unexplored and was discussed by Henry Weiner (Purdue University, USA) who is studying the structure-function relationships of signal peptides, and by Ben de Kruifff (Utrecht, The Netherlands) who is analysing the role of lipids in protein transport. Finally, the understanding of the molecular basis of mitochondrial partitioning and segregation within the cell, and the identification of molecules involved in defining the shape and size of individual mitochondria is a brandnew aspect of mitochondrial biogenesis that is now beginning to flourish (Mike Yaffe). Without any doubt, a lot of interesting developments lie in store for us in the near future.

\section{References}

1 GLICK, B. and SCHATZ, G. (1991) Annu. Rev. Genet. 25, 21-44

2 GLICK, B. S., BEASLEY, E. M. and SCHATZ, G. (1992) Trends Biachem. Sci. $17,453-459$

3 LLLL, R., HERGERSBERC, C., SCHNEIDER, H., SÖLLNER, T, STUART, R. A. and NEUPERT, W. (1992) in Membrane Biogenesis and Protein Targeting (Neupert, W. and Lili, R., eds), pp. 265-276, Elsevier

4 PFANNER, N., SÖLLNER, T. and NEUPERT, W. (1991) Trends Biochem. Sci. 16, 63-67

5 PFANNER, N., RASSOW, I. VAN DER KLEI, I. I. and NEUPERT, W. (1992) Cell 68, 999-1002

6 SECUI-REAL, B., STUART, R. A. and NEUPERT, W. (1992) FEBS Lett. 313, 2-7 